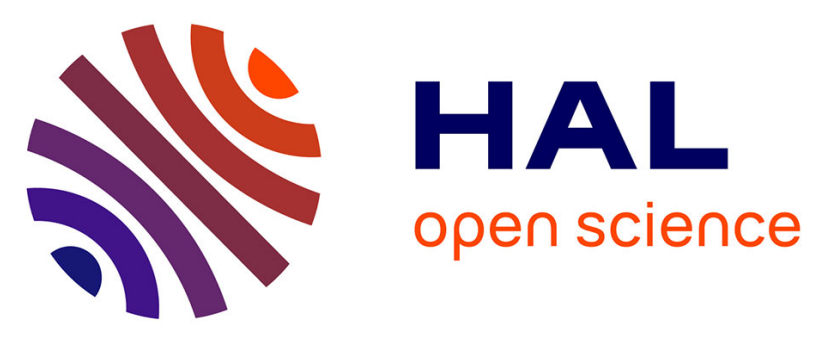

\title{
NMR diffusion measurements under chemical exchange between sites involving a large chemical shift difference
}

Sébastien Leclerc, L Guendouz, A Retournard, D Canet

\section{To cite this version:}

Sébastien Leclerc, L Guendouz, A Retournard, D Canet. NMR diffusion measurements under chemical exchange between sites involving a large chemical shift difference. Concepts in Magnetic Resonance Part A: Bridging Education and Research, 2010, 36, pp.127 - 137. 10.1002/cmr.a.20154. hal01482078

\section{HAL Id: hal-01482078 \\ https://hal.univ-lorraine.fr/hal-01482078}

Submitted on 3 Mar 2017

HAL is a multi-disciplinary open access archive for the deposit and dissemination of scientific research documents, whether they are published or not. The documents may come from teaching and research institutions in France or abroad, or from public or private research centers.
L'archive ouverte pluridisciplinaire HAL, est destinée au dépôt et à la diffusion de documents scientifiques de niveau recherche, publiés ou non, émanant des établissements d'enseignement et de recherche français ou étrangers, des laboratoires publics ou privés.

\section{(1)(1) $\$(0)$}

Distributed under a Creative Commons Attribution - NonCommercial - ShareAlikel 4.0 


\title{
NMR diffusion measurements under chemical exchange between sites
}

\author{
involving a large chemical shift difference
}

\author{
S. Leclerc ${ }^{2)}$, L. Guendouz ${ }^{3)}$, A. Retournard ${ }^{1)}$, D. Canet ${ }^{1)} *$
}

1) Méthodologie RMN (CRM ${ }^{2}$ UMR 7036, Nancy-Université-CNRS), Faculté des Sciences et Techniques, B.P. 239, 54506 Vandoeuvre-lès-Nancy (cedex), France

2) LEMTA (UMR 7563, Nancy-Université-CNRS), BP 160, 54504 Vandoeuvre-lèsNancy (cedex), France

3) Laboratoire d'Instrumentation Electronique de Nancy, BP 239, 54506 Vandoeuvrelès-Nancy (cedex), France

* Corresponding author: Daniel.Canet@rmn.uhp-nancy.fr

\begin{abstract}
$\underline{\text { Abstract }}$
This study concerns the thallium-205 cation in aqueous solution in the presence of a calixarene molecule. While the measurement of the self-diffusion coefficient of pure thallium (without calixarene in the aqueous solution) does not pose any particular problem, major difficulties are encountered with the standard method employing gradient strength increment as soon as thallium is partly complexed by calixarene. With static magnetic field gradients, the NMR signal is so weak that it prevents any reliable measurement whereas radio-frequency (rf) field gradients lead to an unrealistic value of the diffusion coefficient. This failure is explained by the fact that thallium is in fast exchange between two sites (complexed and free thallium) thus exhibiting a single NMR signal although, in the course of the experiment, two signals, with an important difference in resonance frequencies (due to the large thallium chemical shift range), are effectively involved. With the objective to understand these quite unexpected observations, the theory underlying NMR diffusion experiments is first reviewed and criteria of fast exchange are discussed for three parameters: chemical shifts, relaxation rates, diffusion coefficients. It turns out that off-resonance effects are responsible for unwanted defocusing due to rf pulses in the static magnetic field gradient method and for time-dependent gradients in the rf field gradient method. Concerning the latter, a remedy is proposed which consists in applying the stronger gradient and incrementing the gradient pulse durations. After correction for relaxation, the expected value of the diffusion coefficient is retrieved.
\end{abstract}




\section{Introduction}

Self-diffusion coefficients, measured by NMR, are generally achieved under nearly onresonance conditions, meaning that the carrier frequency ( $v_{r}$, the NMR transmitter frequency) is not far from the NMR frequencies $\left(v_{0}\right)$. The issue of a large frequency offset, between $v_{r}$ and $v_{0}$, has been rarely addressed, just because it is usually a simple matter to set $v_{r}$ in the vicinity of all resonance frequencies of interest $\left(v_{0}\right)$. This cannot be evidently the case when fast exchange conditions prevail between two sites of drastically different chemical shifts. We encountered this problem when studying a host-guest system constituted of a thallium cation (the guest) partly complexed by a calixarene molecule (the host). By contrast, when dealing with cesium (as the guest) and the same host, no special difficulty was noticed (1). In both cases, we observe a single signal which is the weighted average between two signals of frequencies $v_{A}$ (corresponding to the complexed cations) and $v_{B}$ (corresponding to the free cations in aqueous solution). This is schematized in Fig. 1. The only difference between cesium and thallium is the extremely broad chemical shift scale of the latter, with the consequence that $\left|v_{A}-v_{B}\right|$ can become very large, and thus that on-resonance conditions can never be achieved for both sites. We thus anticipate some problems either by using static field gradients ( $B_{0}$ gradients) or radio-frequency gradients ( $B_{1}$ gradients) methodologies.

In fact, the measurement of the self-diffusion coefficient of the thallium cation $\left(\mathrm{TlNO}_{3} 0.1 \mathrm{M}\right.$ solution) in aqueous solution was straightforward (with both types of gradients) and we found a value close to the self-diffusion coefficient of water, in agreement with the fact that the cation is surrounded by water molecules. It was however somewhat bewildering to notice that, as soon as calixarene was present, no signal could be observed with $B_{0}$ gradients while, with $B_{1}$ gradients, a result was actually obtained leading however to unrealistic values of the self- 
diffusion coefficient. Obviously, chemical exchange is responsible for such drawbacks and the purpose of the present paper is to decipher the causes of these unexpected observations.

\section{Theory}

It is advisable to go back to the basic principles encompassing diffusion measurements either with $B_{0}$ gradients in the stimulated echo mode (2) (rather than the original PGSE experiment (3) which is not suitable when dealing with short transverse relaxation times, as here) or with the equivalent with $B_{1}$ gradients (4). Both these experiments involve a first gradient pulse which is used to defocus nuclear magnetization according to the location of the molecules bearing the relevant nuclear spins (sometimes, this first gradient pulse is dubbed "encode" pulse). Let us consider a gradient $g$ applied along the $X$ direction of the laboratory frame. It will produce ideally a precession angle ( $B_{0}$ gradients; in the $x, y$ plane of the rotating frame) or a nutation angle ( $B_{1}$ gradients, in the $y, z$ plane if $B_{1}$ is polarized along the $x$ axis of the rotating frame) equal to

$$
\theta(X)=\gamma g X \delta
$$

where $\gamma$ is the thallium gyromagnetic ratio and $\delta$ the duration of the gradient pulse. The component along the $z$ axis is then $\cos [\theta(X)]$. After an interval of duration $\Delta$, called the diffusion interval, a second gradient pulse of the same duration $\delta$ (the "decode" pulse), is applied leading to the same defocusing angle $\theta(X)$. The various time intervals and the angle $\theta(X)$ are defined in Fig. 2.

If the molecules bearing the spins of interest have not moved and if the gradient is timeindependent, of sufficient strength and applied for an appropriate duration so that complete defocusing occurs (that is $\theta(X)$ spans at least $2 \pi$ for all $X$ values existing in the sample), 
the component along $z$ (which ultimately leads to the observed signal) is the average of $\cos ^{2} \theta$, the value of which is $\frac{1}{2}$.

We introduce now diffusion during the interval $\Delta$, so that the angle $\theta(X)$ has to be replaced by $\theta+\varphi$ where $\varphi$ accounts for the fact that the molecule bearing the spins of interest has moved to another location due to translational motions. The average has now to be calculated as

$$
\begin{aligned}
& <\cos \theta \cos (\theta+\varphi)>=<\cos ^{2} \theta \cos \varphi>-<\cos \theta \sin \theta \sin \varphi> \\
& =<\cos ^{2} \theta><\cos \varphi>-(1 / 2)<\sin 2 \theta><\sin \varphi>
\end{aligned}
$$

In [2], it has been assumed that translational motions are independent of the initial location $X$ and this is effectively the case for Brownian motions. If we further assume again that defocusing is complete (and consequently that $<\sin 2 \theta>=0$ ), the preceding expression reduces to

$$
<\cos \theta \cos (\theta+\varphi)>=(1 / 2)<\cos \varphi>
$$

We shall define the usual $q$ variable

$$
q=\gamma g \delta / 2 \pi
$$

With this notation the angle $\varphi$ can be expressed as

$$
\varphi=2 \pi q r
$$

where $r$ is the displacement of the molecule of interest during $\Delta$. The calculation of the mean value of $\cos \varphi$ rests on the well-known distribution function of displacements due to selfdiffusion (5), that is $\frac{1}{\sqrt{4 \pi D \Delta}} \exp \left(-\frac{r^{2}}{4 D \Delta}\right), D$ being the so-called diffusion coefficient

$$
<\cos \varphi>=\frac{1}{\sqrt{4 \pi D \Delta}} \int_{-\infty}^{+\infty} \cos (2 \pi q r) \exp \left(-\frac{r^{2}}{4 D \Delta}\right) d r=\exp \left(-4 \pi^{2} q^{2} D \Delta\right)
$$

In addition to the decay given by [6], we must account for decays due to relaxation and also to diffusion during the gradient pulses. The expression for the latter has been determined in the 
early days of NMR (6) (effect of translational diffusion in the presence of a constant gradient): $\exp \left(-8 \pi^{2} q^{2} D \delta / 3\right)$. Altogether, one has for the observed signal $S(q)$

$$
\begin{aligned}
& S(q)_{B_{0} \text { gradient }} \propto \exp \left(-2 \tau / T_{2}^{e f f}\right) \exp \left(-\Delta / T_{1}\right) \exp \left[-4 \pi^{2} q^{2} D(\Delta+2 \delta / 3)\right] \\
& S(q)_{B_{1} \text { gradient }} \propto \exp \left(-2 \delta / T_{2 \rho}\right) \exp \left(-\Delta / T_{1}\right) \exp \left[-4 \pi^{2} q^{2} D(\Delta+2 \delta / 3)\right]
\end{aligned}
$$

It should be noted that the observed magnetization arises from longitudinal magnetization during $\Delta$, hence the factor $\exp \left(-\Delta / T_{1}\right)$. On the other hand, relaxation during the gradient pulses is accounted for

i) In the case of $B_{0}$ gradients, by the factor $\exp \left(-2 \tau / T_{2}^{\text {eff }}\right), \tau>\delta$ being the total time during which magnetization remains transverse while gradient is applied (including inter-pulse intervals, gradient rise and fall times...). $T_{2}^{\text {eff }}$ is either $T_{2}^{*}$ (simple gradient pulses) or $T_{2}$ (bipolar gradient pulses, see below).

ii) In the case of $B_{1}$ gradients, by the factor $\exp \left(-2 \delta / T_{2 \rho}\right)$ with $1 / T_{2 \rho}=(1 / 2)\left(1 / T_{1}+1 / T_{2}\right)$. With respect to $B_{0}$ gradients, this is especially advantageous, when $T_{2}$ is short and much shorter than $T_{1}$ (about a factor of two is gained, notwithstanding the fact that $\tau>\delta$ ).

In the presence of chemical exchange, the above theory may need to be amended. Indeed, as soon as in 1992, Moonen et al. proposed (GEXSY experiment) to measure the diffusion in exchanging spin systems (7). This was followed by studies of the influence of exchange on DOSY spectra $(8,9)$. An interesting work concerns possible chemical shift modulations in diffusion decays (10). Several applications aimed at determining exchange rates or residence times can be found in the literature (11-13). All these latter works concern however slow exchange (at least with respect to chemical shift). Although a relatively recent publication (14) deals with fast exchange (the topic of the present work), we were unable to find any explanation of our experimental observations in these previous studies. 
As a matter of fact, specific conditions of fast exchange apply to each parameter. This will be now discussed and, for that purpose, we have to define some quantities: $p$, the proportion of complexed thallium, denoted by $A$ in the following; $(1-p)$, the proportion of free Thallium, denoted by $B$ in the following; $\tau_{A}=p \tau$, the residence time in site $A$ with the exchange rate $k$ equal to $1 / \tau ; \tau_{B}=(1-p) \tau$, the residence time in site $B$. We shall consider that fast exchange conditions prevail with respect to a given parameter $G$ if

$$
G_{o b s}=p G_{A}+(1-p) G_{B}
$$

We start with chemical shifts, or, rather, with resonance frequencies. If, between two consecutive data points, the considered spin jumps many times between sites $A$ and $B$, then a weighted average has effectively to be accounted for (see fig. 1), that is

$$
v_{o b s}=p v_{A}+(1-p) v_{B}
$$

As the sampling interval is set for possibly observing $v_{A}$ and $v_{B}$, that is of the order of $1 /\left(2\left|v_{A}-v_{B}\right|\right)$, the condition of fast exchange with respect to chemical shifts can be written as

$$
k>>2\left|v_{A}-v_{B}\right|
$$

Concerning relaxation rates $R$, let us assume (for the sake of simplicity) that they induce, during a time $t$, a magnetization loss equal to $\exp (-R t)$. This means that, at the outcome of the time interval $\left(\tau_{A}+\tau_{B}\right)$, magnetization has decreased by a factor $\exp \left(-R_{A} p \tau\right) \exp \left[-R_{B}(1-p) \tau\right]$. After a first order expansion, we obtain

$$
M\left(\tau_{A}+\tau_{B}\right) \approx M_{0}\left[1-\left(p R_{A}+(1-p) R_{B}\right) \tau\right]+M_{0} p(1-p) R_{A} R_{B} \tau^{2}
$$

Clearly, the first term in the right-hand member of [12] represents a weighted average of relaxation rates, thus the observed quantity in the case of fast exchange (relatively to relaxation rates) provided that the last term is negligible. Consequently, the condition of fast exchange with respect to relaxation rates can be stated as

$$
k>R_{A}, R_{B}
$$


It means that the exchange rate must be greater than the relaxation rates in both sites.

As far as diffusion is concerned, we shall neglect its possible effects during the application of gradient pulses and only consider $\Delta$, the so-called diffusion interval. Therefore, we have just to look at the phase angle $\varphi$ (see eqs. [5] and [6]). Phase angles at the outcome of the intervals $\tau_{A}$ and $\tau_{B}$ are given by (eq. [6])

$$
\begin{aligned}
& \cos \varphi_{A}=\exp \left(-4 \pi^{2} q^{2} D_{A} p \tau\right) \\
& \cos \varphi_{B}=\exp \left[-4 \pi^{2} q^{2} D_{B}(1-p) \tau\right]
\end{aligned}
$$

At the outcome of the time interval $\left(\tau_{A}+\tau_{B}\right)$, the total phase angle $\varphi$ is then

$$
\cos \varphi=\cos \varphi_{A} \cos \varphi_{B}-\sin \varphi_{A} \sin \varphi_{B}=\exp \left[-4 \pi^{2} q^{2} \tau\left(p D_{A}+(1-p) D_{B}\right)\right]
$$

$\sin \varphi_{A}$ and $\sin \varphi_{B}$ are zero because they would be the imaginary part of the Fourier transform of a Gaussian function (eq. [6] is the real part) and it turns out that this Fourier transform is real. Consequently, as can be seen from [15], fast exchange conditions concerning diffusion are met provided that $\Delta$ involves at least one cycle $\left(\tau_{A}, \tau_{B}\right)$ or, in other words, that $\Delta$ is greater than $\left(\tau_{A}+\tau_{B}\right)$. In accord with reference (9), the condition of fast exchange with respect to diffusion coefficients can be expressed as

$$
k>1 / \Delta
$$

Failure of diffusion measurements under chemical exchange involving important offresonance conditions

The usual method for measuring self-diffusion coefficients consists in keeping constant the two intervals $\delta$ and $\Delta$ while repeating the basic experiment with different increments of the gradient strength $g$ so that relaxation decays remain constant. If equations [7] and [8] are valid, the diffusion coefficient is easily deduced from at least two experiments provided that no irreversible defocusing occurs. In these equations, it is however assumed that on-resonance conditions prevail in such a way that defocusing is exclusively produced by a time- 
independent gradient. We show below that exchange processes may rule out the simple view represented by these equations, especially when the two sites exhibit a very important chemical shift difference. As far as our samples are concerned, a chemical shift difference of $110 \mathrm{ppm}$ together with the observation of a single line at $9.4 \mathrm{~T}$ (15) which indicates fast exchange with respect to chemical shifts, ensures also fast exchange with respect of relaxation rates and diffusion coefficients.

\section{Imperfections of radio-frequency pulses}

If the radio-frequency (rf) field has a low amplitude $\left(B_{1}\right)$, off-resonance effects show up in the rotating frame as a tilt of the effective rf field with respect to the polarization axis (say $x$ ), due to a non-negligible $z$ component. The latter is equal (in frequency units) to $v_{0}-v_{r}$, $v_{0}$ being the resonance frequency. This is schematized in figure 3 for the two resonances $v_{A}$ and $v_{B}$ of figure 1 (the amplitude of the $\mathrm{rf}$ field is represented in frequency units by $\left.v_{r f}=\gamma B_{1} / 2 \pi\right)$.

The orientations of effective $B_{1}$ 's are seen to be different for the two resonances $v_{A}$ and $v_{B}$. As a consequence, nutation takes place in different planes and, if the spins move continuously between $v_{A}$ and $v_{B}$ because of chemical exchange, nutation becomes oscillatory. If the duration of the rf pulse encompasses several cycles of exchange, the latter will produce an irreversible defocusing even if $B_{1}$ is homogeneous. This is illustrated by figure 4 where the quality of a $180^{\circ}$ inverting pulse is seen to drop considerably in the case of exchanging thallium.

These considerations concern not only homogeneous rf fields but also rf field gradients. This oscillatory behavior may become detrimental and makes the gradient time-dependent during the gradient pulses. 


\section{$B_{0}$ gradients}

In an attempt to understand the failure of diffusion measurements by $B_{0}$ gradients, we first paid heed to possible effects arising from chemical shift differences between the two sites versus the gradient strength. Let us assume that the resonance frequencies $\left|v_{A}-v_{r}\right|$ and $\left|v_{B}-v_{r}\right|$ (in the rotating frame) are significantly non negligible with respect to $2 \pi \gamma g X$ for all locations $X$ in the sample (see figure 1). This is certainly true in our case, at least, for the first gradient values in experiments dealing with incremented gradients. In such a situation, one must also take into account precession in the rotating frame during the interval $\delta(10)$ and the angle $\theta(X)$ is different for $v_{A}$ and $v_{B}$

$$
\begin{aligned}
& \theta_{v_{A}}(X)=p\left[\gamma g X+2 \pi\left(v_{A}-v_{r}\right)\right] \delta \\
& \theta_{v_{B}}(X)=(1-p)\left[\gamma g X-2 \pi\left(v_{r}-v_{B}\right)\right] \delta
\end{aligned}
$$

Because of fast exchange, we must consider the average of the two angles in [17]. If $v_{r}$ is chosen as in figure $1\left(v_{r}=p v_{A}+(1-p) v_{B}\right)$, this average amounts to $\theta=\gamma g X \delta$ and, contrary to our expectations, nothing particular should occur, in spite of a very large chemical shift difference. As a consequence, if the gradient strength is sufficient, there would be complete defocusing due to the continuous evolution of $X$ across the sample, all values of $\theta$ in the interval $[0,2 \pi]$ having the same probability. Therefore $\langle\cos \theta\rangle_{X}=0$ (defocusing) and $\left\langle\cos ^{2} \theta\right\rangle_{X}=1 / 2$ (refocusing). Moreover, bipolar gradients (two gradient pulses of opposite polarity separated by a $180^{\circ}$ pulse (16)) have been used here (see figure 5 for the details of the sequence). They are known to cancel all chemical shift effects (including those due to the magnetic field inhomogeneity) unless rapid diffusion occurs through background gradients 
As explained above, each rf pulse contributes to an irreversible defocusing. Now, for a commercial spectrometer, thallium is outside the frequency range allowing proper tuning and matching of the probe. As a consequence, the rf field is reduced (thus amenable to the unwanted effects schematized in fig. 3) and owing to the five rf pulses the sequence of figure 5, the NMR signal virtually cancels. As shown in figure 6, instead of $60 \%$ canceled by relaxation, we have observed a loss of more than $90 \%$. Of course, adding a LED subsequence (18) (often used for avoiding ill effects due to eddy currents) with its two additional rf pulses, would still make the situation worse. The higher trace of fig. 6 corresponds to the sequence of figure 5 without any gradient pulse and is therefore indicative of the intrinsic loss by offresonance effects in the course of rf pulses. Conversely if a gradient of moderate strength is applied so that attenuation by diffusion is negligible, we expect a loss by a further factor of two (refocusing by the second gradient). This is shown by the lower trace of fig. 6 . Although running a diffusion experiment as in fig. 6 seems somewhat challenging, we attempted to verify that gradients work as they should do even in the presence of exchange. Unfortunately, as expected from fig. 6 , these experiments failed and we were unable to observe any decay due to diffusion. This poor signal-to-noise ratio can be easily understood on the basis of irreversible defocusing due to the off-resonance effects detailed above. As a matter of fact, accounting for the large chemical shift difference experienced by thallium in the two sites (110 ppm), the tilt angle between $v_{1 \text { eff }}$ and the $x$ axis (see fig. 3 ) is, in our case (with a $90^{\circ}$ pulse of $40 \mu \mathrm{s}$ ), of the order of $60^{\circ}$ whereas a proper tuned probe (with a $90^{\circ}$ pulse of $10 \mu \mathrm{s}$ ) would only reduce this angle to $30^{\circ}$ (a value still too high). The remedies are therefore obvious: i) improve the quality of the probe so as to increase significantly the rf field strength, ii) go to lower static magnetic field so as to reduce the frequency difference $\left|v_{A}-v_{B}\right|$. Indeed, similar experiments performed at $4.7 \mathrm{~T}$ with a dedicated probe (albeit without $B_{0}$ gradients, not available on this instrument) exhibit the above mentioned effects to a much lesser extent. 


\section{$B_{1}$ gradients}

The actual sequence is depicted in fig. 7 and is seen to involve only one rf pulse (which is simply a read-pulse). Contrary to the above experiments with $B_{0}$ gradients and, as shown in fig. 8, a signal is actually observed for exchanging thallium but this signal leads eventually to an unexpectedly large diffusion coefficient (thus to a decay faster than expected) when the experiment is carried out by incrementing the gradient strength. Although this fast decay occurs essentially at low gradient strength values (see figure 9), the explanation is quite different from the one invoked for $B_{0}$ gradients. As shown previously, because of exchange, one switches continuously between two effective $B_{1}$ fields of different directions. This renders the $B_{1}$ gradient oscillatory and therefore time dependent (denoted by $g(t)$ in the following). As a consequence, the expression of $\theta(X)$ becomes

$$
\theta(X)=\int_{0}^{\delta} \gamma g(t) X d t
$$

It is thus equivalent to consider that the gradient is constant and that it is $X$ which experiences these oscillations and, therefore, to consider it as a sort of translational diffusion motion. Accordingly, it may be necessary to account for diffusion during the application of gradient pulses with an effective diffusion coefficient, $D_{\text {eff }}$, presumably much longer than the intrinsic diffusion coefficient and which obviously depend i) on the exchange rate, ii) on $\left|v_{A}-v_{B}\right|$. Anyway, for low values of $q$, equation (8) must be rewritten as

$$
S(q)_{B_{1} \text { gradient }} \propto \exp \left(-2 \delta / T_{2 \rho}\right) \exp \left(-\Delta / T_{1}\right) \exp \left(-4 \pi^{2} q^{2} D \Delta\right) \exp \left(-8 \pi^{2} q^{2} D_{\text {eff }} \delta / 3\right)
$$

Thus, the initial decay being faster than expected, the measured diffusion coefficient (from a fit of the low quality data of fig. 9) is necessarily larger than the true diffusion coefficient. 


\section{A possible remedy: radio-frequency field gradient with increments of the gradient pulse duration}

As seen above, off-resonance effects constitute the main problem when dealing with fast exchange involving large chemical shift differences. This problem can be circumvented by a sufficient rf field strength or, within the $B_{1}$ gradient methodology, by a sufficient gradient strength. This evidently rules out the gradient strength increment method and suggests the use of a strong gradient throughout the experiment. Two possibilities can therefore been envisioned: either incrementing $\Delta$, or incrementing $\delta$. The former requires to be corrected for the $T_{1}$ attenuation during $\Delta$ and, in fact, due to the duration of this interval, we found that $T_{1}$ attenuation largely dominates the attenuation due to diffusion. This possibility was therefore left aside. The latter possibility works very well when $T_{2 \rho}$ is much larger as compared to any value of $\delta$ (as this is the case for $\mathrm{TINO}_{3}$ solution, see figure 10). Of course, for shorter $T_{2 \rho}$, it is less straightforward but, sensitivity permitting, the experiment should be successful provided that the decay curve is corrected for $T_{2 \rho}$ relaxation effects ( $T_{2 \rho}$ can be deduced from $T_{1}$ and $T_{2}$ which have to be measured beforehand). This has been verified by the experiments of fig. 10 .

In spite of scattered data (due a modest signal-to-noise ratio and to a finite gradient value which may not totally prevent the off-resonance effects), the decay concerning exchanging thallium leads to a correct value for the diffusion coefficient $\left(1.710^{-5} \mathrm{~cm}^{2} \mathrm{~s}^{-1}\right)$ which should be the weighted average between free and complexed thallium, the latter being identical to the one of the host molecule (independently measured). The expected value $\left(1.610^{-5} \mathrm{~cm}^{2} \mathrm{~s}^{-1}\right.$, calculated from these data and from the known proportion of complexed thallium) is sufficiently close to the experimental determination for demonstrating the validity and the potentiality of the proposed method. 


\section{Experimental}

$B_{0}$ gradient experiments have been performed with a Bruker Avance DRX400 spectrometer equipped with a TBIZ probe normally operating, for X nuclei, in the $18-162 \mathrm{MHz}$ frequency range. It was nevertheless possible to tune this probe for the Thallium-235 resonance frequency $(230.8 \mathrm{MHz}$ ), at the expense of deteriorated performances (see above). With this instrument, the maximum gradient strength is $30 \mathrm{G} \mathrm{cm}^{-1}$ and, in practice (sine shaped gradient pulses), is reduced to $18 \mathrm{G} \mathrm{cm}^{-1}$.

$B_{1}$ gradient experiments have been performed with a home-made $4.7 \mathrm{~T}$ spectrometer, equipped with a probe dedicated to the observation of thallium-235 (114.8 MHz) and possessing a $B_{1}$ gradient coil system capable of delivering a maximum gradient of $11 \mathrm{G} \mathrm{cm}^{-1}$. This probe, shown in fig. 11, follows from a new design by which the uniformity and strength of rf gradients are considerably improved (19).

\section{Conclusion}

Through this study, off-resonance effects have been shown to constitute the major issue when dealing with diffusion experiments in the presence of fast chemical exchange involving a large difference in resonance frequencies between the two sites involved in this exchange process. Although thallium may seem particular due to its important chemical shift scale, the problem is in fact of general concern because such a situation may be encountered with more common nuclei at higher static magnetic field values. When using $B_{0}$ gradients, the difficulties lie in a severe sensitivity loss and, paradoxically, the remedy would be to go to lower static magnetic field values so as to reduce difference in resonance frequencies between the two sites. On the other hand, the $B_{1}$ gradient methodology using gradient pulse 
increments seems also quite viable, again at lower field, provided that sufficiently strong gradients are available. Our new instrumental design (19) should fulfill this requirement. 


\section{References}

1. Cuc D, Canet D, Morel J.P, Morel-Desrosiers N, Mutzehardt P. 2007. ${ }^{133}$ Cs diffusion NMR spectroscopy: a tool for probing metal cation- $\pi$ interactions in water. Chem. Phys. Chem. 8:643-645

2. Tanner JE.1970. Use of the stimulated echo in NMR diffusion studies. J Chem Phys 52:2523-2526.

3. Stejskal EO, Tanner JE. 1965. Spin diffusion measurements: spin echoes in the presence of a time-dependent field gradient . J Chem Phys 42 :288-292.

4. Canet D. 1997. Radiofrequency field gradient experiments. Prog Nucl Magn Reson Spectrosc 30:101-135.

5. Price WS. 1997. Pulsed-field gradient nuclear magnetic resonance as a tool for studying translational diffusion. Part I. basic theory. Concepts Magn Reson 9:299-336.

6. Torrey HC. 1956. Bloch equations with diffusion terms. Phys Rev 104:563-565.

7. Moonen CTW, van Gelderen P, Vuister GW, van Zijl PCM. 1992. Gradient-Enhanced Exchange Spectroscopy. J Magn Reson 97:419-425.

8. Johnson, Jr. CS. 1993. Effects of chemical exchange in diffusion-ordered 2D NMR spectra. J Magn Reson A 102:214-218.

9. Cabrita EJ, Berger S, Bräuer P, Kärger J. 2002. High-resolution DOSY with spins in different chemical surroundings: influence of particle exchange. J Magn Reson 157:124-131. 10. Chen A, Johnson Jr, CS, Lin M, Shapiro MJ. 1998. Chemical exchange in diffusion NMR experiments. J Am Chem Soc 120:9094-9095.

11. Liu M, Toms HC, Hawkes GE, Nicholson J.K., Lindon J.C. 1999. Determination of the relative NH proton lifetimes of the peptide analogue viomycin in aqueous solution by NMRbased diffusion measurement. J Biomol NMR 13:25-30. 
12. Cabrita EJ, Berger S 2002. HR-DOSY as a new tool for the study of chemical exchange phenomena. Magn Reson Chem 40:S122-S127.

13. Thureau P, Ancian B, Viel S, Thévand A. 2006. Determining chemical exchange rates of the uracil labile protons by NMR diffusion experiments. Chem Comm 2:200-202.

14. Gottwald A, Creamer LK, Hubbard PL, Callaghan PT. 2005. Diffusion, relaxation, and chemical exchange in casein gels: a nuclear magnetic resonance study. J Chem Phys 122:034506.

15. Cuc D, Bouguet-Bonnet S, Morel-Desrosiers N, Morel JP, Mutzenhardt P, Canet D. 2009. Behavior of cesium and thallium cations inside a calixarene cavity. Evidendence of cation- $\pi$ interaction in water. J Phys Chem B 113: 10800-10807.

16. Cotts RM, Hoch MJR, Sun T, Marker JT. 1989. Pulsed field gradient stimulated echo methods for improved NMR diffusion measurements in heterogeneous systems. J Magn Reson 83:252-266.

17. Kuntz JF, Trausch G, Palmas P, Mutzenhardt P, Canet D. 2007. Diffusive diffraction phenomenon in a porous polymer material observed by NMR using radio-frequency field gradients. J Chem Phys 126:134904.

18. Gibbs SJ, Johnson Jr, CS. 1991. A PFG NMR experiment for accurate diffusion and flow studies in the presence of eddy currents. J Magn Reson 93:395-402.

19. Guendouz L, Leclerc S, Retournard A, Hedjiedj A, Canet D. 2008. Single-sided radiofrequency field gradient with two unsymmetrical loops: Application to nuclear magnetic resonance. Rev Scient Instrum 79:123704. 
Figure captions

Figure 1: The carrier frequency $\left(v_{r}\right)$ is supposed to coincide with the observed signal (solid line) which arises, by chemical exchange, from the two lines at frequencies $v_{A}$ and $v_{B}$ : $v_{r}=p v_{A}+(1-p) v_{B}$, where $p$ is the proportion of complexed thallium.

Figure 2. Definition of the time intervals and of the angle by which the nuclear magnetization at a position $X$ is rotated by the application of a gradient pulse (in the rotating frame). Left: $B_{0}$ gradient. Right: $B_{1}$ gradient ( $B_{1}$ being assumed to lie along $x$ ).

Figure 3. Off-resonance effects expressed in frequency units. $v_{\text {leff }}$ standing for $\gamma B_{1}^{\text {eff }} / 2 \pi$ provides the amplitude and direction of the effective rf field.

Figure 4. $230.8 \mathrm{MHz}^{205} \mathrm{Tl}$ NMR spectra $(9.4 \mathrm{~T}) .128$ scans. Duration of the $90^{\circ}$ pulse: $43 \mu \mathrm{s}$. The positive peak is obtained after a $90^{\circ}$ read-pulse. The negative peak results from the application of the same read-pulse immediately after a supposed $180^{\circ}$ pulse. Left: aqueous solution of $\mathrm{TINO}_{3} 0.1 \mathrm{M}$; the inversion rate is $73 \%$. Right: exchanging thallium; the inversion rate is $55 \%$. All data concerning these two samples can be found in ref. (15). $p=0.2$ for the sample of exchanging thallium.

Figure 5. Details of the STE_BP sequence (Stimulated Echo with Bipolar Pulses)

Figure 6. $230.8 \mathrm{MHz}$ NMR spectra (9.4 T) of exchanging thallium (25530 Hz between sites) obtained through the sequence of figure $5(\delta=2 \mathrm{~ms}, \Delta=200 \mathrm{~ms}) .2000$ transients. Blue trace: gradients set to zero. Red trace: with gradients of $0.3 \mathrm{G} / \mathrm{cm}$.

Figure 7. Details of the diffusion sequence with rf gradients (or $B_{1}$ gradients).

Figure 8. $114.8 \mathrm{MHz}$ NMR spectrum (4.7 $\mathrm{T}$; duration of the $90^{\circ}$ pulse: $11.5 \mu \mathrm{s}$ ) of exchanging ${ }^{205}$ Thallium $(12765 \mathrm{~Hz}$ between sites) obtained through the sequence of figure 7 without gradients ( $\delta=0.5 \mathrm{~ms}, \Delta=200 \mathrm{~ms}$ ). 512 transients.

Figure 9. $114.8 \mathrm{MHz} B_{1}$ gradient diffusion experiments by incrementing the gradient strength. Left: $\mathrm{TINO}_{3} 0.1 \mathrm{M}$ solution decay ( $\Delta=300 \mathrm{~ms} ; \delta=5 \mathrm{~ms}$ ) leading to a diffusion coefficient of $1.910^{-5} \mathrm{~cm}^{2} \mathrm{~s}^{-1}$ (which is the expected value, close to water diffusion coefficient). Right: exchanging thallium decay $(\Delta=200 \mathrm{~ms} ; \delta=2 \mathrm{~ms})$ leading to a diffusion coefficient of $9.710^{-5}$ $\mathrm{cm}^{2} \mathrm{~s}^{-1}$ (a totally unrealistic value).

Figure 10. 114.8 $\mathrm{MHz} B_{1}$ gradient diffusion experiments by incrementing the gradient pulse duration $\delta\left(\Delta=200 \mathrm{~ms} ; \mathrm{g}=10.28 \mathrm{G} \mathrm{cm}^{-1}\right)$. Left: $\mathrm{TlNO}_{3} 0.1 \mathrm{M}$ solution decay leading to a diffusion coefficient of $1.910^{-5} \mathrm{~cm}^{2} \mathrm{~s}^{-1}$. Right: exchanging thallium decay leading to a diffusion coefficient of $1.710^{-5} \mathrm{~cm}^{2} \mathrm{~s}^{-1}$. 
Figure 11. The $B_{1}$ gradient assembly operating (normally in an upright position) at 114.8 MHz. The saddle shaped coil is used for normal rf pulses and signal detection. The two loops, closer to the saddle shaped coil, are the actual gradient coil. The third loop (top) serves as a power transmitter through its inductive coupling to the gradient coils. 


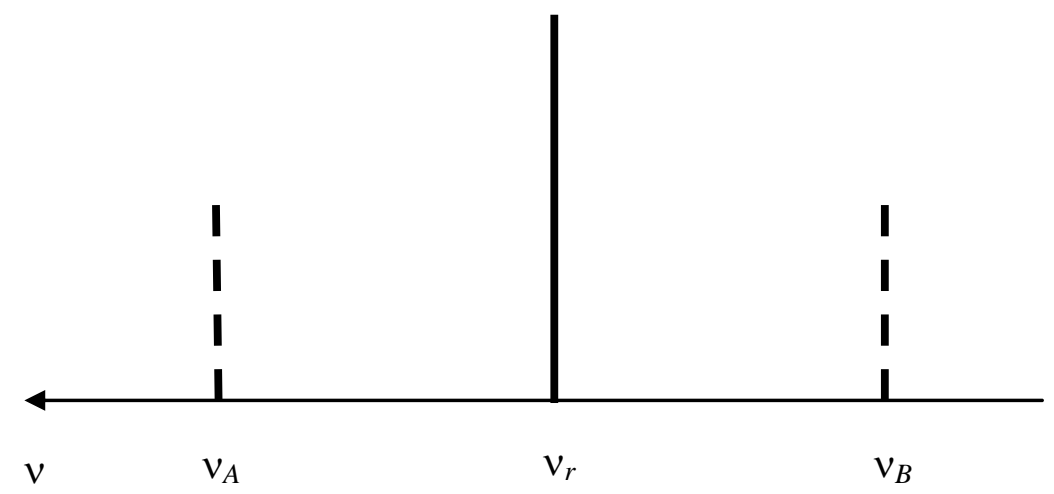

Figure 1 

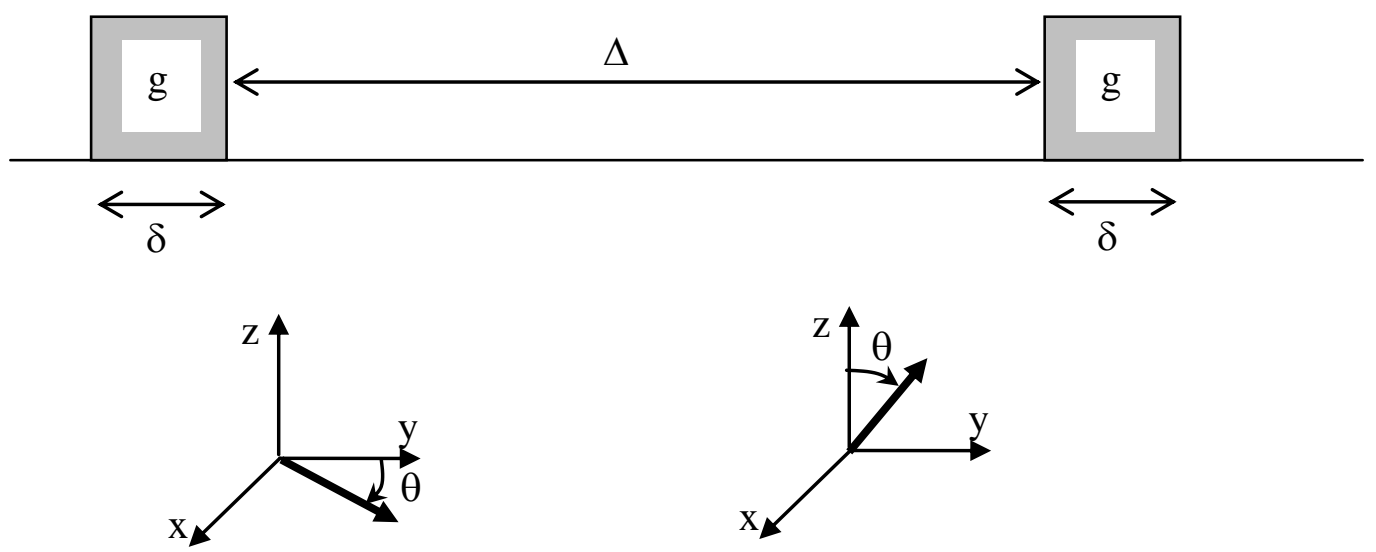

Figure 2 

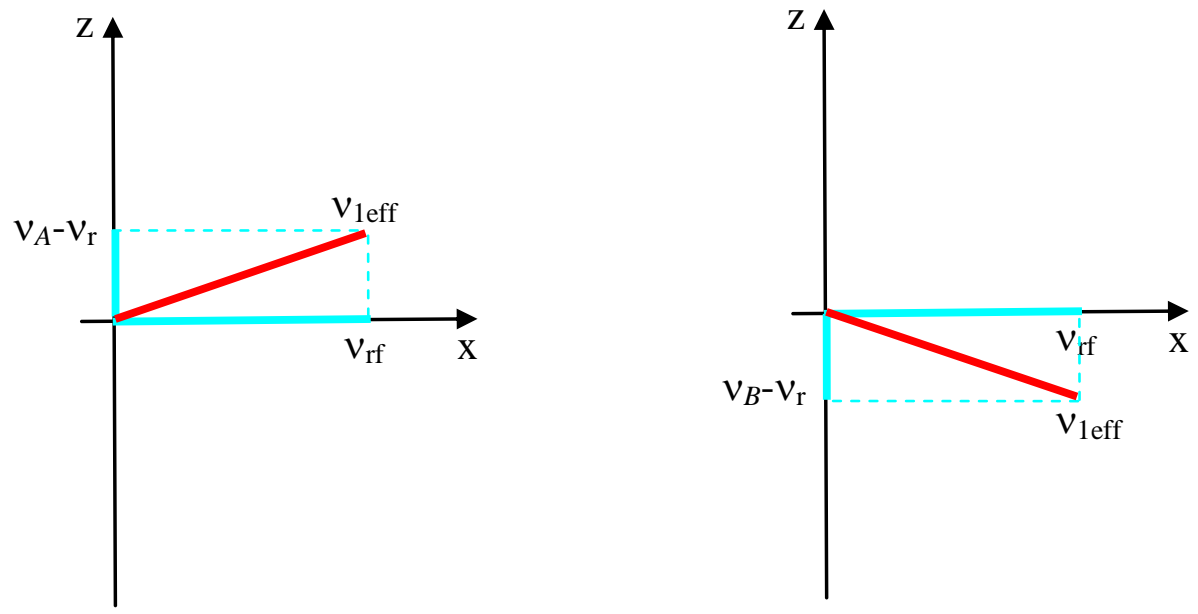

Figure 3 

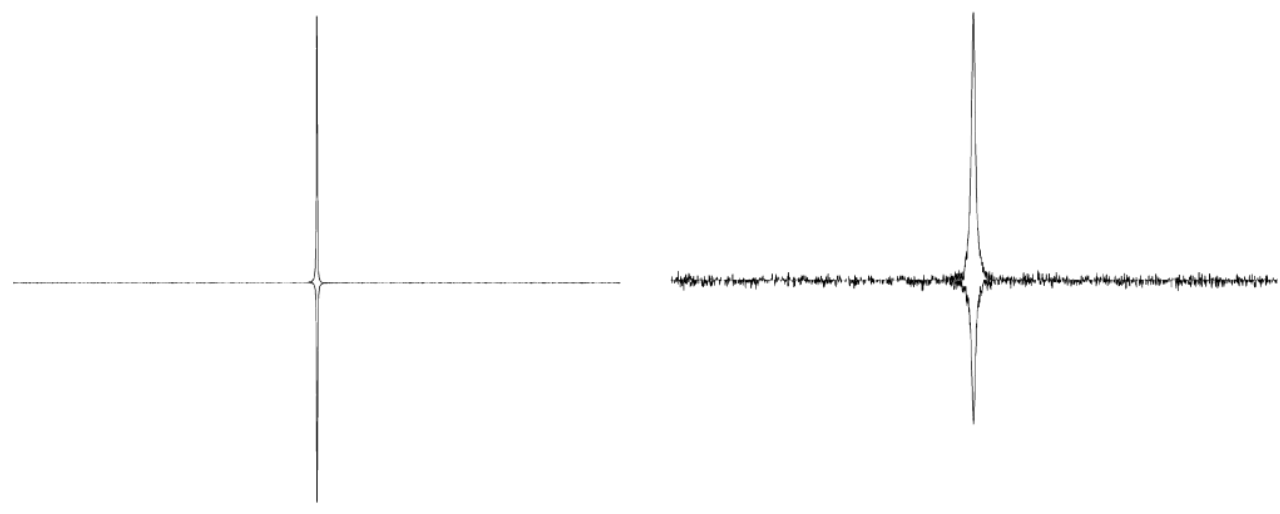

Figure 4 


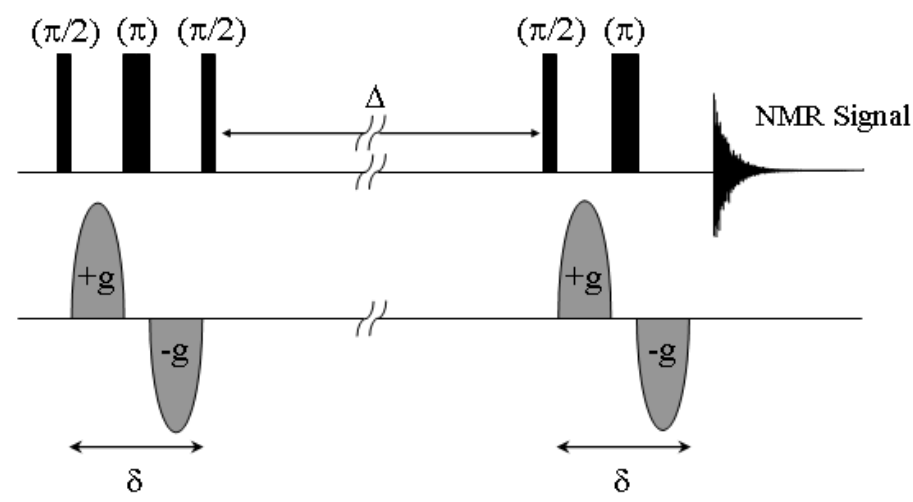

Figure 5 


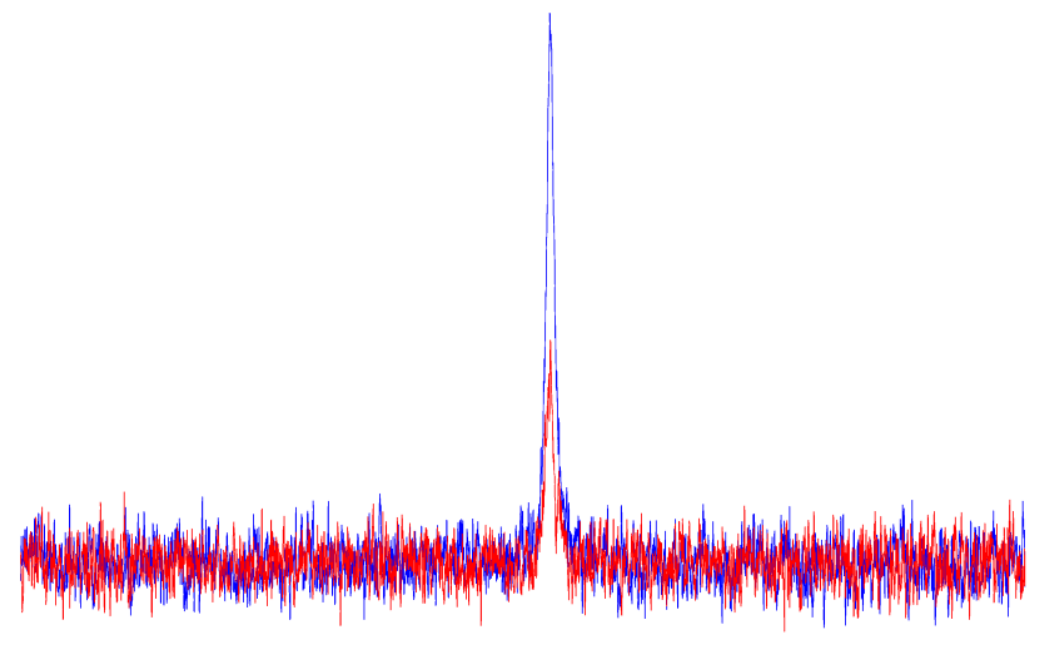

Figure 6 


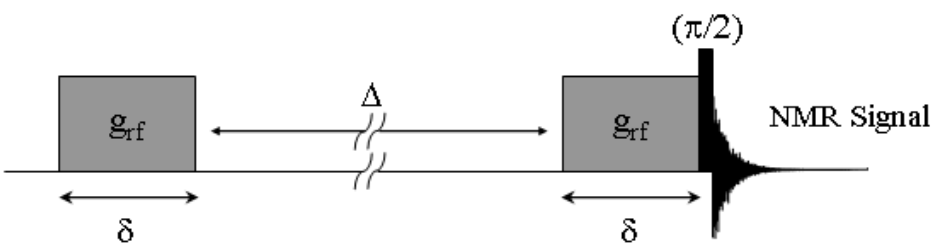

Figure 7 
Figure 8 

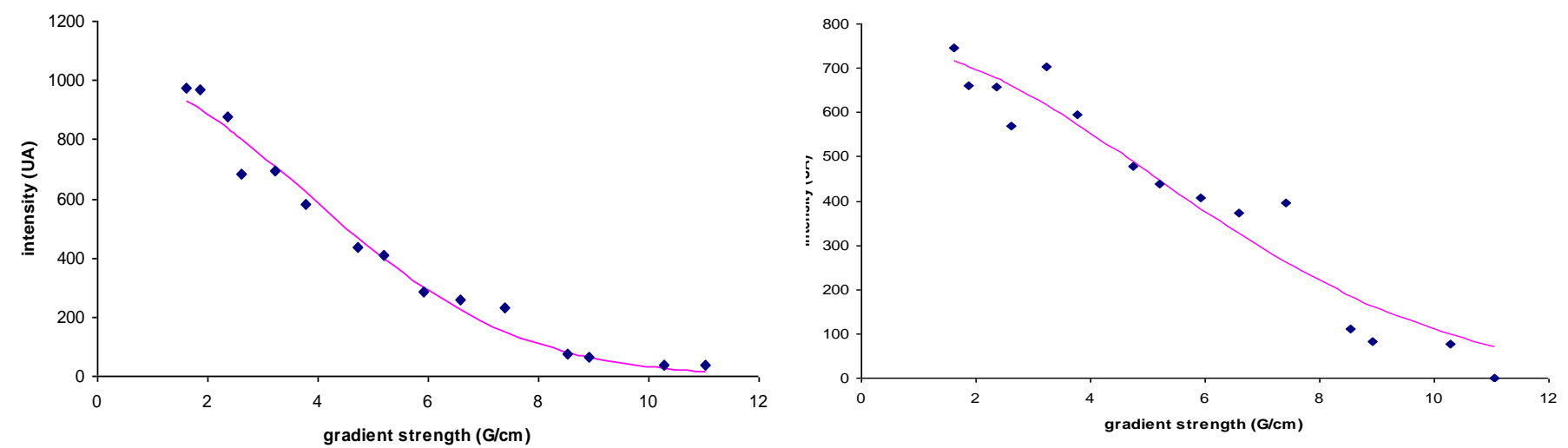

Figure 9 

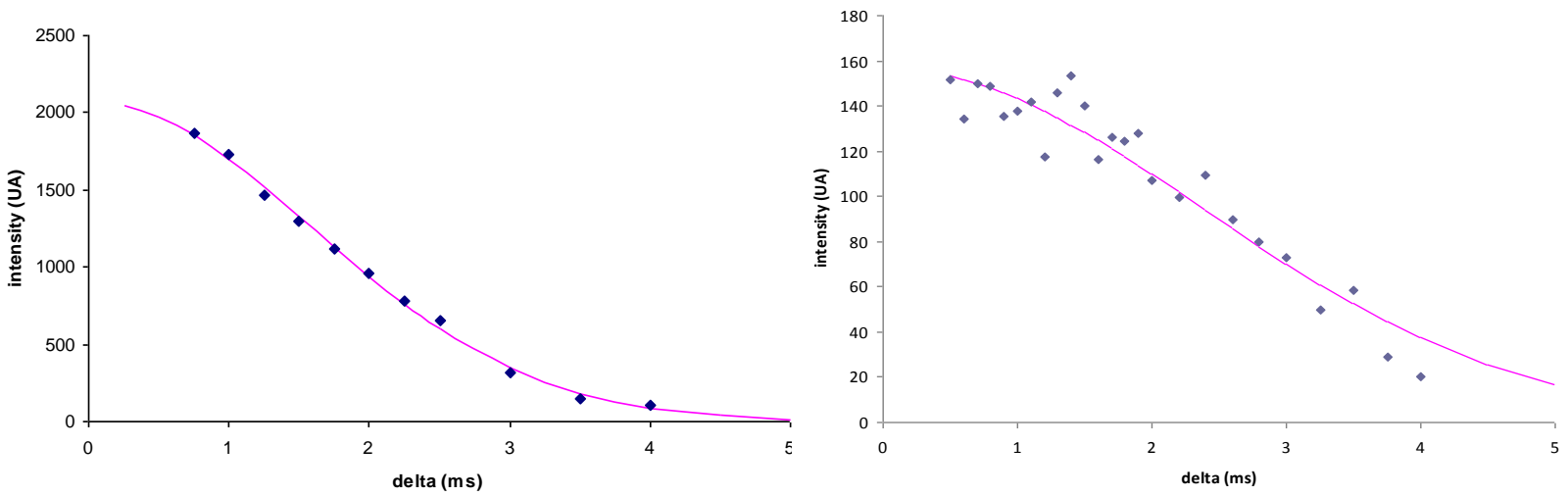

Figure 10 


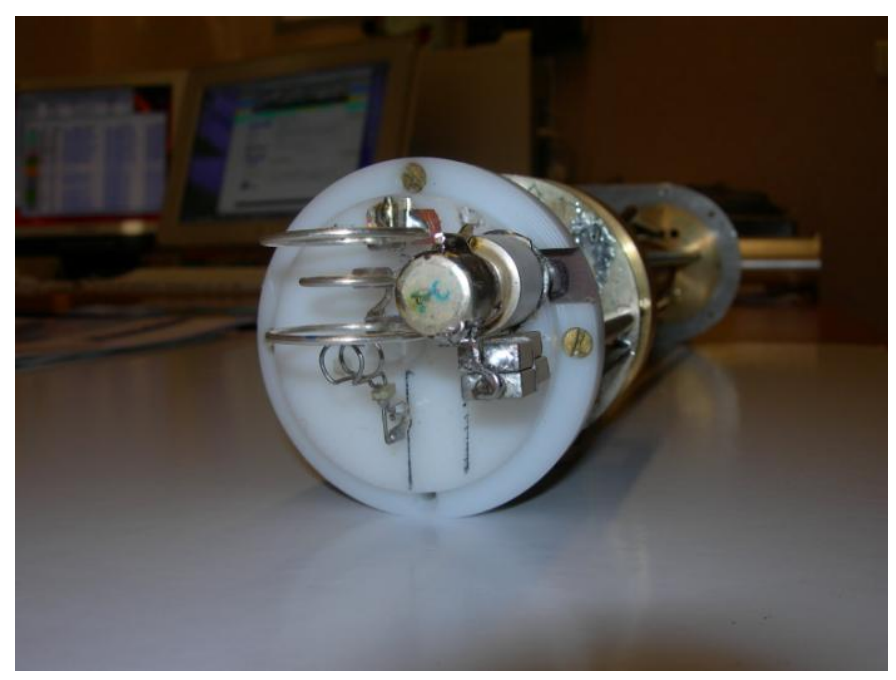

Figure 11 